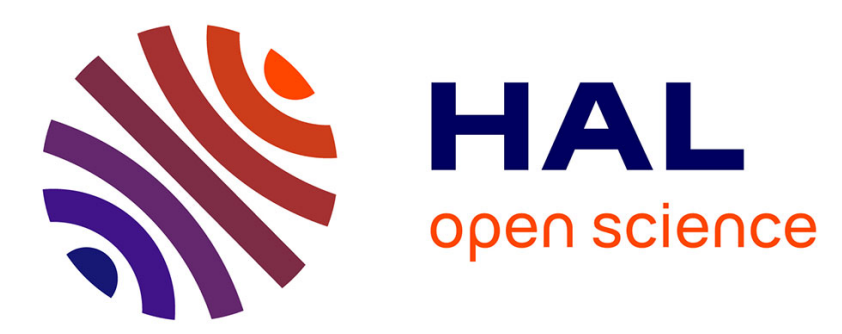

\title{
Using water activity measurements to evaluate rheological consistency and structure strength of sludge
}

G. Agoda Tandjawa, E. Dieudé Fauvel, R. Girault, J.C. Baudez

\section{To cite this version:}

G. Agoda Tandjawa, E. Dieudé Fauvel, R. Girault, J.C. Baudez. Using water activity measurements to evaluate rheological consistency and structure strength of sludge. Chemical Engineering Journal, 2013, 228, p. 799 - p. 805. 10.1016/j.cej.2013.05.012 . hal-00916411

\section{HAL Id: hal-00916411 \\ https://hal.science/hal-00916411}

Submitted on 10 Dec 2013

HAL is a multi-disciplinary open access archive for the deposit and dissemination of scientific research documents, whether they are published or not. The documents may come from teaching and research institutions in France or abroad, or from public or private research centers.
L'archive ouverte pluridisciplinaire HAL, est destinée au dépôt et à la diffusion de documents scientifiques de niveau recherche, publiés ou non, émanant des établissements d'enseignement et de recherche français ou étrangers, des laboratoires publics ou privés. 


\title{
Using water activity measurements to evaluate rheological consistency and structure strength of sludge
}

\author{
G. Agoda-Tandjawa, E. Dieudé-Fauvel*, R. Girault. and J.-C. Baudez \\ Irstea, UR TSCF, Domaine des Palaquins - Montoldre, F-03150, France
}

E-mail of the corresponding author: emilie.dieude-fauvel@irstea.fr

Tel.: +33470477429

\begin{abstract}
In this study, an original method of characterization of water/solid matter interactions in sewage sludge has been developed, based on both rheological characteristics and thermodynamic water activity determinations, in order to check whether a link could be made with water activity and its mechanical properties. The effect of solid matter content, flocculation and ageing time on water activity and rheological parameters has been investigated. Through this study, we showed that rheological parameters ( $G$ ' and $G$ ' ') of both raw and flocculated sewage sludge at optimal dose of polymer increase with solids concentration following a power-law, whereas in the same way water activity decreases following an exponential relationship (Arrhenius dependence). A slight increase of water activity values and those of G' and G' moduli with added polymer was also highlighted. On the other hand, we have shown that during ageing, the rheological parameters $G^{\prime}$ and $G$ ', decreased upon increasing the ageing time whereas in the same way, water activity increases. Rheological parameters clearly evidenced thermodynamic water activity dependence, regarding solid concentration and ageing time, with a decrease of G' and G' upon increasing water activity. Thus, water/solid matter interactions were supposed to intervene importantly in the rheology of sewage sludge. From all these results, it appears clearly that water activity measurements can be regarded as an effective and easy tool for evaluating the state of structuration of sewage sludge and for predicting its structural, textural and mechanical properties during dewatering and ageing.
\end{abstract}

Keywords: rheology; water activity; sewage sludge; network strength, flocculation; dewatering; ageing

\section{Introduction}

Nowadays sludge management is of major concern due to environmental pressure and economic considerations. Optimizing treatment process is a great challenge and an accurate estimation of sludge rheological properties and sludge structure strength is required for the 
design of pumping systems or for the control of the dewatering steps [1]. Slatter [2-5] has also consistently shown that rheology plays a fundamentally important role in analyzing the hydrodynamic behavior of the sludge, as it flows in pipes and reactors. However, several papers underlined that consistency and strength of the structure are not directly linked to solid content [6-9]: the qualitative composition of sludge is of much greater importance than quantitative composition (i.e. basic solid content). This is related to the presence of extracellular polymeric substances (EPSs) of different nature, depending on the type of sludge and the influence of sludge treatment. EPS are highly charged polymers that interact with water in a similar way as gels [10-13], influencing the strength of the structure regarding the nature of interactions between solids and water which can be balanced between steric and electrostatic [35]

As a consequence, great attention has been paid to water repartition within sludge. For example, extensive studies, largely reviewed by Vaxelaire and Cézac [14], have been performed to estimated water states within activated sludge by using several techniques such as : differential scanning calorimetry (DSC) [15], dilatometry [16, 17], drying [16], combined thermal gravimetry analysis and differential thermal analysis [18], nuclear magnetic resonance spectroscopy [19], centrifugal settling [20] and desorption isotherms [21]. Based on these studies, four categories of water in sludge were classically distinguished:

- Free water, which represents the largest part of the sludge. Thermodynamically, it behave as pure water which is not associated with solid particles and including void water not affected by capillary forces [22].

- Interstitial water, which is trapped inside crevices and interstitial spaces of flocs and organisms [17, 22, 23].

- Surface (or vicinal) water, which held onto the surface of solid particles by adsorption and adhesion [17, 22, 23].

- Bound (or hydration) water [17, 22, 23].

Besides this classification and considering the impact of the polymeric matrix which constitutes activated sludge, Mikkelsen and Keiding [12] have introduced the term "waterholding" to cover both surface bound water and osmotic water as well as water trapped within the polymer network. Thus, sewage sludge can be seen as soft matter and improving knowledge on water/solid matter interactions is one of the clues to improve sludge rheology, especially network strength which plays a fundamental work in conditioning and dewatering 
[36] In addition, as rheological properties of soft matter are strongly related to interactions among particles dispersed in medium $[24,25]$, it is not surprising that the state of water could appear as a relevant parameter to evaluate sludge consistency and structure strength.

One can easily relate the way water is linked to solid matter to its fugacity. Fugacity is the escaping tendency of a substance and the activity of species is defined as the ratio between the fugacity $f$ and the escaping tendency of a pure material, $f_{0}$ [37]: $A=f / f_{0}$. When dealing with water, a $w$ subscript is designated for the substance: $A_{w}={ }^{f} / f_{0} \cdot A_{w}$ is defined as the activity of water, or the escaping tendency of water in the considered system divided by the escaping tendency of pure water (with no radius of curvature). As the state of water in suspensions arises from interactions between water molecules and solid matter in the medium, coupling water activity with rheological properties will thus be an attempt to obtain information on the effects of water/solid mater interactions on the rheological properties of sewage sludge and the strength of its structure.

Thus, this work focuses on the characterization of the water/solid matter interactions, based on water activity measurement and on the sludge rheological properties, in order to check whether a link could be made with sludge structure. By focusing on the impact of solid concentration, conditioning and ageing time, we show that rheological properties and water activities can be connected together and that water activity may be used as a quick and easyto-use tool to evaluate strength of sludge structure.

\section{Materials and methods}

\subsection{Materials}

Activated sludge was sampled at the Varennes-sur-Allier waste water treatment plant (Allier, France). The samples had a pH between 6.8 and 7.2, conductivity between 1105 and $1240 \mathrm{Scm}^{-1}$ and dry matter content between $1.22 \% \mathrm{w} / \mathrm{w}$ and $1.35 \% \mathrm{w} / \mathrm{w}$. After sampling, sludge was gently concentrated to $1.9 \% \mathrm{w} / \mathrm{w}$ by decantation and stored at $4{ }^{\circ} \mathrm{C}$ for maximum 3 days, to reduce temporal variability effect of biochemical composition change (fermentation).

As described later, for experiments, sludge was thickened with and without conditioning.

For sludge conditioning, structured cationic polyacrylamide in emulsion form (polymer FLOPAM EM 840 from SNF SAS product range, Andrézieux, France) was used. This 
conditioning polymer was also sampled at the Varennes-sur-Allier waste water treatment plant. Polymer solutions $(47.53 \% \mathrm{w} / \mathrm{w})$ were diluted at $0.63 \% \mathrm{w} / \mathrm{w}$ in deionized water under gentle stirring at room temperature for $6 \mathrm{~h}$, according to instructions given by the manufacturer (SNF SAS, Andrézieux, France). Thus, polymer solutions were prepared at least $24 \mathrm{~h}$ prior to application as described by Saveyn et al.[26].

\subsection{Sludge conditioning}

A Stuart Scientific Jar Test device was used. The procedure was as follows: 200 rpm during 2 min for intense mixing of the polymer into the sludge, followed by a 8 min slow stirring period at $30 \mathrm{rpm}$ to promote floc growth. Half a liter of sludge $(1.43 \% \mathrm{w} / \mathrm{w})$ was mixed with different doses of polymer. The optimum dose of polymer was determined with capillary suction time (CST) procedure, using a Triton Electronics $304 \mathrm{M}$ CST meter. Each measurement was performed five times.

In this work, sludge was flocculated at the defined optimum dose of polymer. After conditioning, the samples were drained with a coffee filter during $4 \mathrm{~h}$.

\subsection{Thickening and ageing tests}

Sludge was divided into several identical samples, then thickened in a laboratory ultracentrifuge, at a fixed temperature and fixed duration but at different velocities corresponding to centrifugal accelerations ranging from 4000 to $12000 \mathrm{~g}$ for the raw sludge and from 2000 to $14000 \mathrm{~g}$ for the flocculated sludge. The operating conditions are the following:

- time of centrifugation: $5 \mathrm{~min}$

- temperature: $20^{\circ} \mathrm{C}$

- bowl: designed for 6 tilted tubes of unit volume of $40 \mathrm{ml}$.

After centrifugation, solids concentrations were determined by weighting the dry residue after drying at $105^{\circ} \mathrm{C}$ for $24 \mathrm{~h}$. The corresponding solids concentrations of the thickened samples are reported in Table 1.

After thickening, sludge was stored at $4^{\circ} \mathrm{C}$ to limit fermentation. Before experiments, sample was first acclimatized for about $40 \mathrm{~min}$ in a water bath at $20^{\circ} \mathrm{C}$. Another separate sample (at $10.5 \%$ solids $)$ was stored at room temperature $\left(20^{\circ} \mathrm{C}\right)$ during 16 days to focus on the impact of ageing. 
Note that centrifuge may alter sludge structure and modify rheological and water activity parameters but same considerations will be made with mechanical dewatering or soft drying. Thus, in the following, we assume that thickening sludge by short time centrifugation will not affect the results.

\subsection{Rheological measurements}

Dynamic rheological measurements were carried out using MCR 300 and MCR 301 (Anton Paar Physica) controlled stress rheometers equipped with a $50 \mathrm{~mm}$ parallel plates geometry and a Peltier temperature controller. The gap was fixed at $1 \mathrm{~mm}$ for the raw sludge and at 2 $\mathrm{mm}$ for the flocculated sludge. Moreover, to prevent evaporation during measurements, samples were covered with paraffin oil.

Before each measurement, raw sludge was first homogenized at $300 \mathrm{rpm}$ during $5 \mathrm{~min}$ with a moderated intensity mixer (IKA RW20 Digital Dual-Range Mixer, IKA-Werke GmbH \& Co. KG, Staufen, Germany). Then samples were poured onto the rheometer plate at $20^{\circ} \mathrm{C}$ and slowly squeezed between the plans and finally left at rest for $10 \mathrm{~min}$. Note that flocculated sludge was not subjected to shear melting to avoid deflocculation.

After that, sludge was submitted to a frequency sweep, from 0.1 to $100 \mathrm{rads}^{-1}$ at a constant shear strain in the linear viscoelastic region (LVE), fixed at $0.3 \%$. To ensure that viscoelastic measurements were carried out in the LVE domain, strain sweep experiments were conducted from $0.01 \%$ to $10 \%$. For all the experiments, $0.3 \%$, was found to be in the linear region. Each measurement was performed in triplicate and results are presented in the form of G' (storage modulus) and G" (loss modulus) as functions of the frequency.

\subsection{Water activity measurements}

Water activity was determined with a computer-controlled Rotronic HygroLab 2 (Rotronic AG, Bassersdorf, Switzerland). It operates from 0.5 to $50^{\circ} \mathrm{C}$. The sample is placed in a sealed container and slowly exchanges moisture with the air inside the container until equilibrium is reached. The equilibration process is monitored by measuring the humidity of the air above the sample with a relative humidity sensor.

Because temperature is a crucial factor, the whole measuring system (probes, disposable sample cups, chamber of the Rotronic HygroLab 2) and the product samples are 
placed in an incubator set at the desired temperature. The incubator is controlled by a thermostated water bath.

Two probes were used simultaneously. Each probe measures the relative humidity at a given (controlled) temperature, which gives water activity of the sample.

Prior to measurements, disposable sample cups were filled up to 4/5 (approximately with $38 \mathrm{~g}$ of sample to be measured), with the cover on. Then, the cups were placed into the chamber of the Rotronic HygroLab 2 and kept to rest during $10 \mathrm{~min}$ as for rheological measurements. Five minutes before the end of the time of rest, the covers were removed and the probes were immediately putted on top of the sample holder. The data (current water activity and temperature values) were recorded at the end of the time of rest. Each measurement was performed in 6-fold. We are aware this procedure did not give us the water activity at the equilibrium but regarding the high fermentable nature of sludge, waiting of the equilibrium (more than $24 \mathrm{~h}$ regarding [14]) would have induce an experimental bias: we could not have considered sludge would have been the same from the beginning to the end of the measurement. Thus, we intentionally consider the measurement of the relative humidity after 5 minutes by using the AwQuick mode of the Rotronic Hygrolab 2 as an acceptable estimation of water activity.

According to the supplier, the AwQuick mode accelerates the measurement of water activity and provides a result in typically 5 minutes. When temperature conditions are stable (both at the product and probe), the measurement obtained with the AwQuick mode is generally within $+/-0.005$ aw of the measurement that would be obtained by waiting for full equilibrium of the product and probe.

The same procedure as in rheological measurements was followed to homogenized samples prior to measurements.

\section{Results and discussion}

\subsection{Capillary suction time}


When the polymer dose increased from 0.007 to $0.107 \mathrm{~g} / \mathrm{kg}$ sludge dry matter, CST values decrease from $\sim 44 \mathrm{~s}$ to $\sim 9 \mathrm{~s}$ and then increase with further increasing of the dose (Fig. 1). The minimum in the CST-profile allows the determination the point at which polymer overdosing starts. This point was defined as the optimum polymer dose which is in accordance with previous studies [27, 28].

\subsection{Viscoelastic properties and water activity dependence with solid concentration}

Both raw and flocculated sewage sludge exhibited solid viscoelastic behavior, whatever the solids concentrations (Fig. 2). The loss tangent value ( $\left.\tan \delta=\mathrm{G}^{\prime}{ }^{\prime} / \mathrm{G}^{\prime}\right)$, was of the order of 0.1 leading samples to gel-like structure (Baudez et al., 2013). Moreover, storage moduli, G', and loss moduli, G', increased with an increase in the solids concentration of both raw and flocculated sludge throughout the range of applied frequencies, indicating a more rigid network when the solid concentration increases. In addition, whether sludge was flocculated or not, the rheological parameters G' and G' at 1 rads $^{-1}$ increased upon increasing the solids concentration (from $1.9 \% \mathrm{w} / \mathrm{w}$ to $12.78 \% \mathrm{w} / \mathrm{w}$ for the raw sewage sludge; and from $8.78 \%$ $\mathrm{w} / \mathrm{w}$ to $15.82 \% \mathrm{w} / \mathrm{w}$ for the flocculated sludge), following a power-law of the form:

$$
G=a \cdot\left(C-C_{O}\right)^{n}
$$

where $C_{0}$ is the lowest concentration below which there is no structure.

The slopes of the curve are close, with 3.35 and 3.6 for raw and flocculated sludge respectively (Fig. 3). This relative constant slope confirms that, whether sewage sludge was flocculated or not, the medium is structured in a similar way.

Moreover, addition of polymer at optimal dose of sewage sludge basically increases the value of storage and loss moduli throughout the whole solids concentration range $(8.78 \% \mathrm{w} / \mathrm{W}$ - $15.82 \% \mathrm{w} / \mathrm{w})$, meaning that the viscoelastic properties of the sludge were reinforced with flocculation. Such a result was already reported by Chen et al.[29] who showed that shear complex modulus of conditioned sludge, $\mathrm{G}^{*}$, increased to an extent of 2 or 3 orders of magnitude compared to untreated sludge (1.5 order of magnitude in our case, which may be explained by the nature of the polymer). According to Chen et al.[29], the reinforcement of the viscoelastic properties by adding polymer can be related to the fact that the adsorbed polymer may therefore provide enough inter or intra-attractive forces, e.g. van der Waals force or via hydrogen bonding, in addition to electrostatic forces between the oppositely charged polymer and the initially negatively charged sludge particles. 
In parallel, in our range of concentration, water activity decreased upon increasing the solid concentration for both raw and flocculated sludge, following kind of an Arrhenius law (Fig. 4):

$$
A_{w}=1-\exp \left(-\frac{\alpha}{C-C_{0}}\right)
$$

where $C_{0}$ is the lowest concentration below which there is no structure and as a consequence, water activity must be equal to 1 . By definition, $\mathrm{C}$ is the same than in (1) and it is what we found experimentally (table 2).

Similar trend was already observed for bound water in sewage sludge during dewatering [30-32].

Furthermore, one can notice that flocculation tends to increase water activity values (fig. 4), indicating that at the same concentration, water is becoming more accessible when polymer is added. This is in agreement with the literature in which an increase of the amount of free water is reported when some chemical conditioners are added to sludge [16, 19, 30, 31]. To explain this behavior, these papers suggested that chemical conditioners act either by replacing water molecules adsorbed on the particle surface or by affecting the water binding capacity to the particle $[16,31]$. Chu and Lee [33] agreed with this explanation when the dose of conditioner is below the charge neutralisation point. Our results show that this explanation is also valid at the optimal polymer dose.

\subsection{Viscoelastic properties and water activity dependence during ageing}

Similar mechanical spectra as in Fig. 2 have been obtained for raw sludge during ageing (Fig. 5). The storage moduli $\left(G^{\prime}\right)$ and the loss moduli $\left(G^{\prime \prime}\right)$ decreased with the ageing time throughout the range of applied frequencies. The rheological parameters $G^{\prime}$ and $G^{\prime \prime}$ at 1 rads $^{-1}$ decrease when ageing time increases (Fig. 6), following an inverse relationship with ageing time:

$$
G \sim \frac{1}{t}
$$

Such a decrease of rheological parameters was already observed and explained by Baudez and Coussot [7] who showed that during storage, organic carbohydrate polymers are hydrolysed and transformed into volatile fatty acids. This change in solids composition was also highlighted by [34].

In parallel, water activity increases (Fig. 7), following a power-law of the form of:

$$
A_{w}=1-\varepsilon \cdot\left(t-t_{0}\right)^{\beta}
$$


This increase of water activity could indicate that during ageing, some water is released from flocs, but it is inconsistent with the hydrolysis phenomena which induce a consumption of water. We assume this increase of water activity is the consequence of volatile fatty acid synthesis. Because they are amphiphilic materials, they organise themselves to limit contact with water and consequently, water becomes less bounded to the solids.

To summarize, when the solid concentration increases, rheological characteristics increase while water activity decreases and when fermentation process occurs, rheological characteristics decrease while water activity increase. Moreover increase of the solid concentration implies a strengthening of the solid structure while fermentation induces a weakening of this structure. Then these results allow us to draw some trends between rheological solid-like characteristics and water activity (Fig. 8 and 9) and then to consider water activity as an indicator of the strength of the solid structure: at a given solid concentration, the smaller the water activity, the stronger the network structure.

\section{Conclusions}

In this study, an original method of characterization of water/solid matter interactions has been developed, based on thermodynamic water activity determination and on the sludge rheological properties, in order to check whether a link could be made with sludge structure and its mechanical properties. The effect of solid matter content, flocculation and ageing time on water activity and rheological parameters has been investigated. Through this study, we showed that rheological parameters (G' and G') of both raw and flocculated sewage sludge at optimal dose of polymer increase with solids concentration following a power-law, whereas in the same way water activity decreases following an exponential relationship (Arrhenius dependence). Addition of polymer at optimal dose of sewage sludge basically increases the values of storage and loss moduli and those of water activity throughout the whole solids concentration range. This was related to the effects of adsorbed polymer on sludge particles. Rheological parameters clearly evidenced thermodynamic water activity dependence with a decrease of G' and G', upon increasing water activity. It appears clearly that, besides solids particle-particle interactions, water/solid matter interactions contribute strongly in the rheology of both raw and flocculated sludge. On the other hand, we have also shown that during ageing, the rheological parameters G' and G', decreased upon increasing the ageing 
time, whereas in the same way, water activity increases. This result means that the change of sludge composition due to fermentation impacts water fugacity and consequently the rheological properties. Furthermore, the rheological parameters ( $G$ ' and $G$ ') also clearly evidence water activity dependence during ageing, with a decrease of storage modulus $G^{\prime}$ and loss modulus G' upon increasing water activity.

From all these results, it appears clearly that water activity measurements can be regarded as an effective and easy tool for evaluating the network strength of sewage sludge and for predicting its structural, textural and mechanical properties during dewatering and ageing. Further researches need to be done in the case of other various domestic and industrial sludge in which it could be possible to establish a distribution of water states into different categories. Moreover, coupling rheological measurements and near infrared spectroscopy (NIR spectroscopy) could also be an original interesting approach, as we are now doing, to deepen the knowledge of moisture distribution in various sludge; NIR spectroscopy being able to analyse change in water bonding.

Acknowledgements: The authors wish to thank Jean-Claude Megnien and Philippe Héritier for their help in activated sludge sampling. We are also grateful for the financial support provided by Region Auvergne (France).

\section{References}

[1] S.K. Dentel, Evaluation and role of rheological properties in sludge management, Water Science and Technology, 36 (1997) 1-8.

[2] P. Slatter, Pipe flow of highly concentrated sludge, Journal of Environmental Science and Health Part a-Toxic/Hazardous Substances \& Environmental Engineering, 43 (2008) 15161520 .

[3] P.T. Slatter, The rheological characterisation of sludge, Water Science and Technology, 36 (1997) 9-18.

[4] P.T. Slatter, Sludge pipeline design, Water Science and Technology, 44 (2001) 115-120.

[5] P.T. Slatter, The hydraulic transportation of thickened sludge, Water Sa, 30 (2004) 614616.

[6] P. Battistoni, G. Fava, F. Cecchi, P. Pavan, Rheology of sludge from semi-dry anaerobic digestion of municipal solid waste, Environmental Technology, 12 (1991) 897-905.

[7] J.C. Baudez, P. Coussot, Rheology of aging, concentrated, polymeric suspensions: Application to pasty sewage sludge, Journal of Rheology, 45 (2001) 1123-1140.

[8] V. Lotito, L. Spinosa, G. Mininni, R. Antonacci, The rheology of sewage sludge at different steps of treatment, Water Science and Technology, 36 (1997) 79-85.

[9] L. Spinosa, K. Wichmann, Sludge characterization: the role of physical consistency, Water Science and Technology, 49 (2004) 59-65. 
[10] K. Keiding, L. Wybrandt, P.H. Nielsen, Remember the water - A comment on EPS colligative properties, in, 2001, pp. 17-23.

[11] Y. Liu, H.H.P. Fang, Influences of extracellular polymeric substances (EPS) on flocculation, settling, and dewatering of activated sludge, Critical Reviews in Environmental Science and Technology, 33 (2003) 237-273.

[12] L.H. Mikkelsen, K. Keiding, Physico-chemical characteristics of full scale sewage sludge with implications to dewatering, Water Research, 36 (2002) 2451-2462.

[13] I.W. Sutherland, Exopolysaccharides in biofilms, flocs and related structures, Water Science and Technology, 43 (2001) 77-86.

[14] J. Vaxelaire, P. Cézac, Moisture distribution in activated sludge: A review, Water Research, 38 (2004) 2214-2229.

[15] D.J. Lee, S.F. Lee, Measurement of bound water content in sludge: The use of differential scanning calorimetry (DSC), Journal of Chemical Technology and Biotechnology, 62 (1995) 359-365.

[16] J. Robinson, W.R. Knocke, Use of dilatometric and drying techniques for assessing sludge dewatering characteristics, Water Environment Research, 64 (1992) 60-68.

[17] J.K. Smith, P. Aarne Vesilind, Dilatometric measurement of bound water in wastewater sludge, Water Research, 29 (1995) 2621-2626.

[18] G.W. Chen, W.T. Hung, I.L. Chang, S.F. Lee, D.J. Lee, Continuous classification of moisture content in waste activated sludge, Journal of Environmental Engineering, 123 (1997) 253-258.

[19] J. Bower Carberry, R.A. Prestowitz, Flocculation effects on bound water in sludge as measured by nuclear magnetic resonance spectroscopy, Applied and Environmental Microbiology, 49 (1985) 365-369.

[20] D.J. Lee, Measurement of bound water in waste activated sludge: Use of the centrifugal settling method, Journal of Chemical Technology and Biotechnology, 61 (1994) 139-144.

[21] J. Vaxelaire, P. Mousques, J.M. Bongiovanni, J.R. Puiggali, Desorption isotherms of domestic activated sludge, Environmental Technology, 21 (2000) 327-335.

[22] P.A. Vesilind, The role of water in sludge dewatering, Water Environment Research, 66 (1994) 4-11.

[23] K.R. Tsang, P.A. Vesilind, Moisture distribution in sludge, Water Science and Technology, 22 (1990) 135-142.

[24] A. Mourchid, A. Delville, J. Lambard, E. Lécolier, P. Levitz, Phase diagram of colloidal dispersions of anisotropic charged particles: Equilibrium properties, structure, and rheology of laponite suspensions, Langmuir, 11 (1995) 1942-1950.

[25] F. Pignon, A. Magnin, J.M. Piau, Thixotropic colloidal suspensions and flow curves with minimum: Identification of flow regimes and rheometric consequences, Journal of Rheology, 40 (1996) 573-587.

[26] H. Saveyn, G. Pauwels, R. Timmerman, P.V.D. Meeren, Polyelectrolyte conditioning effect in sludge electrodewatering, Filtration, 8 (2008) 220-226.

[27] E. Dieudé-Fauvel, S.K. Dentel, Sludge conditioning: Impact of Polymers on Floc structure, Journal of Residuals Science and Technology, 8 (2011) 101-108.

[28] H. Saveyn, S. Meersseman, O. Thas, P. Van Der Meeren, Influence of polyelectrolyte characteristics on pressure-driven activated sludge dewatering, Colloids and Surfaces A: Physicochemical and Engineering Aspects, 262 (2005) 40-51.

[29] B.H. Chen, S.J. Lee, D.J. Lee, Rheological characteristics of the cationic polyelectrolyte flocculated wastewater sludge, Water Research, 39 (2005) 4429-4435.

[30] F. Colin, S. Gazbar, Distribution of water in sludge in relation to their mechanical dewatering, Water Research, 29 (1995) 2000-2005. 
[31] N. Katsiris, A. Kouzeli-Katsiri, Bound water content of biological sludge in relation to filtration and dewatering, Water Research, 21 (1987) 1319-1327.

[32] C.C. Wu, C. Huang, D.J. Lee, Bound water content and water binding strength on sludge flocs, Water Research, 32 (1998) 900-904.

[33] C.P. Chu, D.J. Lee, Moisture distribution in sludge: Effects of polymer conditioning, Journal of Environmental Engineering, 125 (1999) 340-345.

[34] S.G. Pavlostathis, E. Giraldo-Gomez, Kinetics of anaerobic treatment: A critical review, Critical Reviews in Environmental Control, 21 (1991) 411-490.

[35] J.C. Baudez, R.K. Gupta, N. Esthiaghi, P. Slatter, The viscoelastic behaviour of raw and anaerobic digested sludge: Strong similarities with soft-glassy materials. Water Research, 47 (2013) 173-180.

[36] B. Ormeci, M.M Abu-Orf, Protocol to measure network strength of sludges and its implications for dewatering, Journal of Environmental Engineering, 131 (2005) 80-85.

[37] C. van Den Berg, S. Bruin, Water activity and its estimation in food systems: theoretical aspects. In water activity: influences on food quality. Eds L.B. Rockland and G.F. Stewart, New York academic press (1981) 1-64.

\section{List of figures}

Table 1: Solids concentrations of raw and flocculated sludge after thickening at different centrifugal accelerations 
Solids concentrations

Centrifugal accelerations

$(\%, w / w)$

(g)

Raw sludge

Flocculated sludge

\begin{tabular}{ccc}
\hline 2000 & 1.9 & 8.78 \\
3000 & - & 8.81 \\
4000 & 3.2 & 10.28 \\
5000 & 5.28 & 10.48 \\
6000 & 6.28 & 11.56 \\
7000 & 6.58 & - \\
8000 & - & 13.16 \\
9000 & 9.60 & 14.00 \\
10000 & 10.71 & - \\
12000 & 12.78 & 15.44 \\
13000 & - & 15.60 \\
14000 & - & 15.82 \\
\hline
\end{tabular}

Table 2 : Fitting parameters of Equation (1) and (2)

\begin{tabular}{|c|c|c|c|c|}
\hline & \multicolumn{2}{|c|}{ Raw sludge } & \multicolumn{2}{c|}{ Conditioned sludge } \\
\hline & $\mathrm{G}^{\prime}$ & $\mathrm{G}^{\prime}$ & $\mathrm{G}^{\prime}$ & $\mathrm{G}^{\prime}$ \\
\hline $\mathrm{a}$ & 4.001 & 0.437 & 3.000 & 0.321 \\
\hline $\mathrm{C}_{0}$ & 0.232 & 0.238 & 0.370 & 0.370 \\
\hline $\mathrm{n}$ & 3.356 & 3.331 & 3.609 & 3.607 \\
\hline$\alpha$ & & & & \\
\hline $\mathrm{C}_{0}$ & 24.27 & & 27.756 & \\
\hline
\end{tabular}




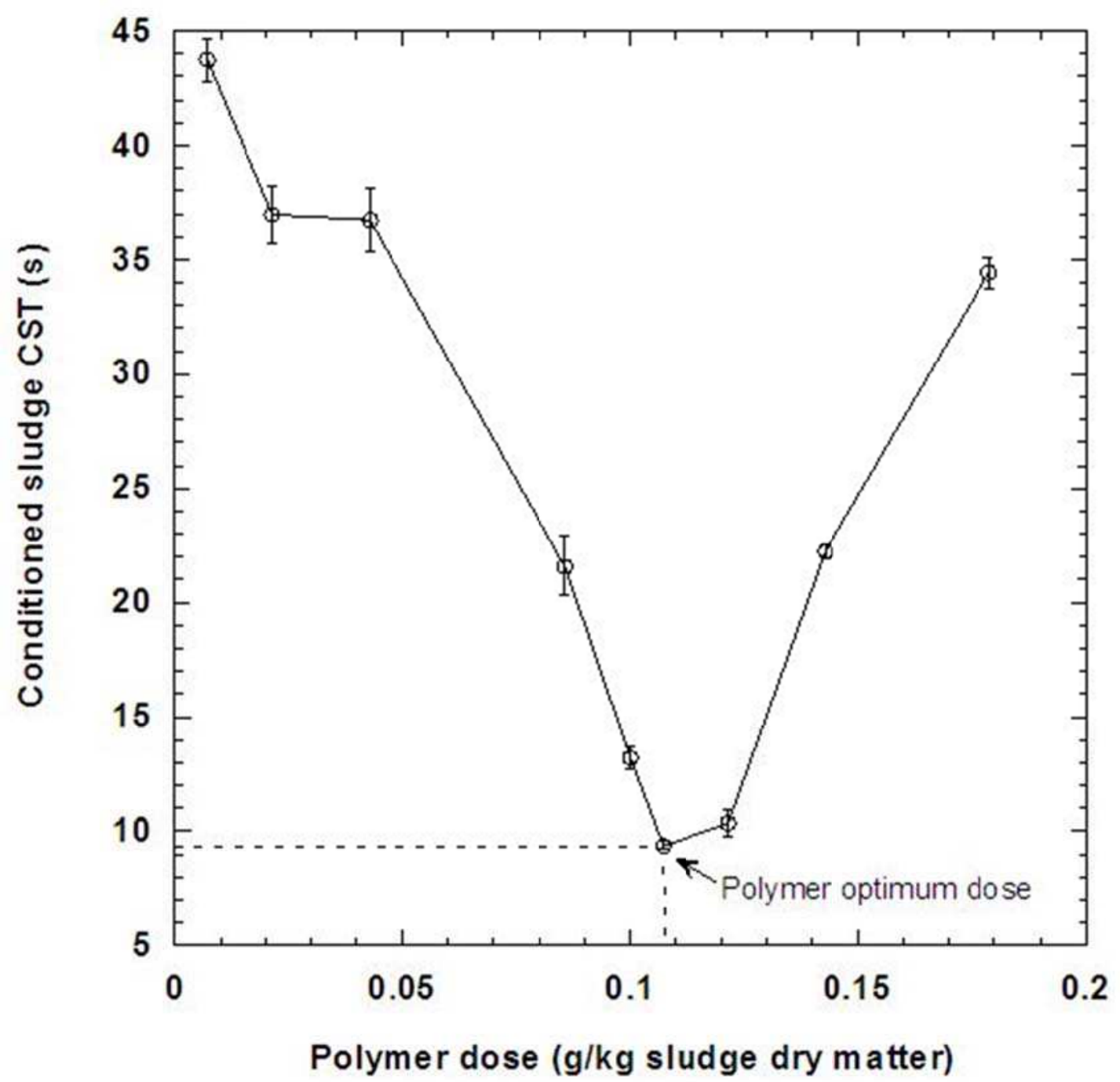

Figure 1: Capillary suction time (CST) as a function of polymer dose 

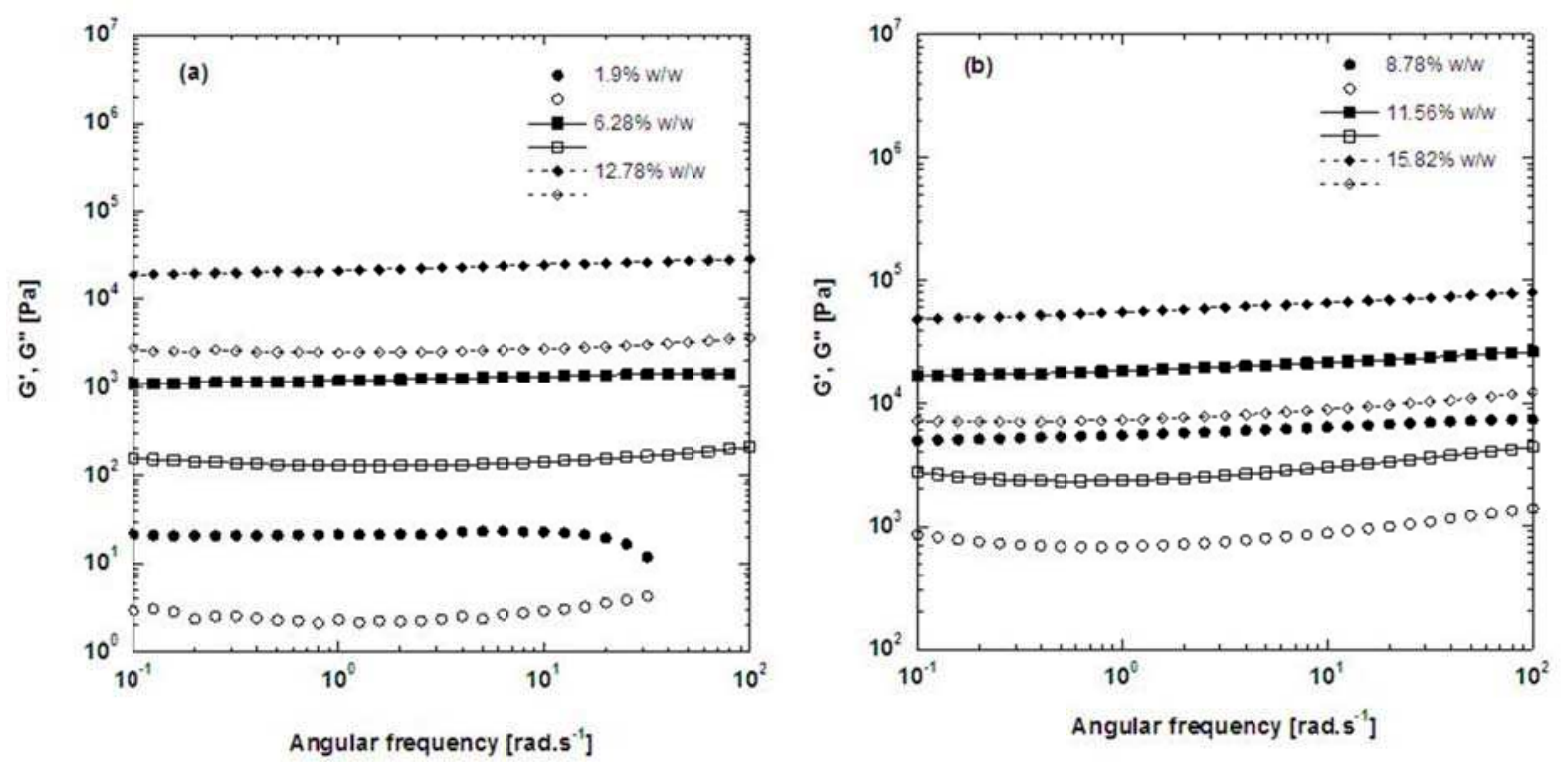

Figure 2: Mechanical spectra of raw (a) and flocculated (b) sewage sludge at various solids concentrations after dewatering. Measurement temperature, $20^{\circ} \mathrm{C}$; strain, $0.3 \%$; time of rest before measurement, 10 min. G', filled symbols; G', empty symbols

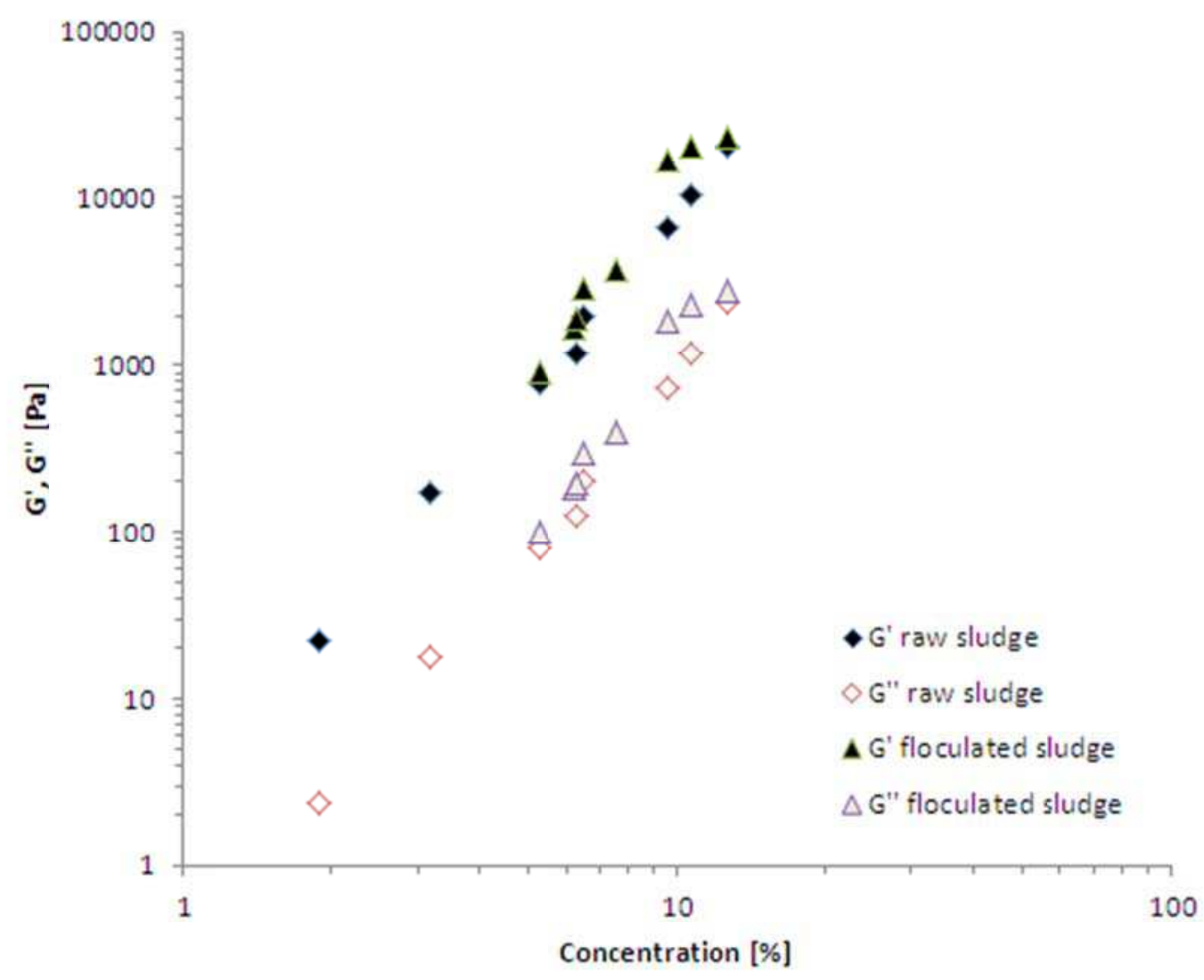

Figure 3: Storage modulus G' and loss modulus G" as a function of raw and flocculated sludge solids concentration after dewatering, at $20^{\circ} \mathrm{C}$ and at an angular frequency of $1 \mathrm{rads}^{-1}$. The parameters of the power law are summarized in table 2 


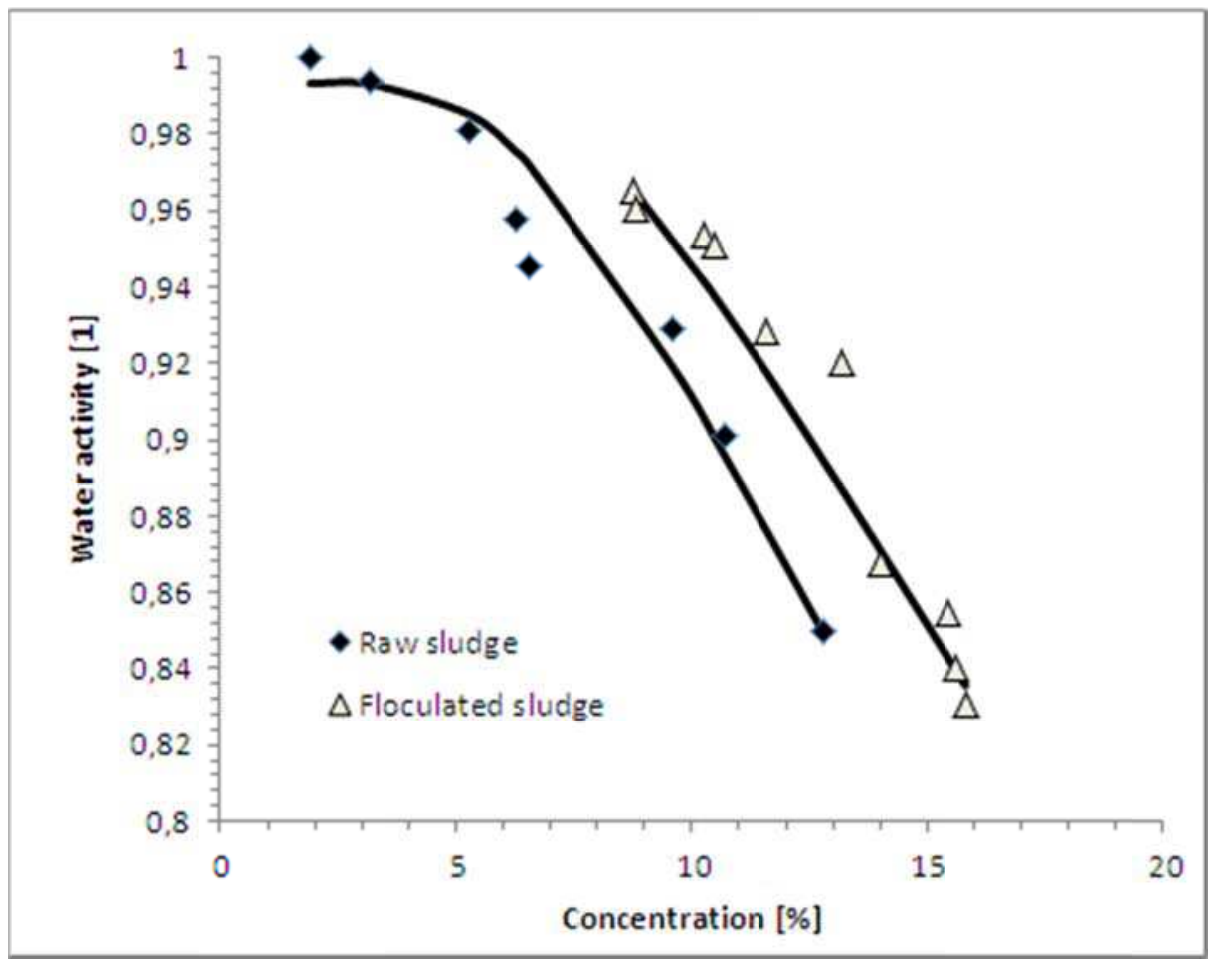

Figure 4: Water activity, Aw as a function of raw and flocculated sludge solids concentration after dewatering, at $20^{\circ} \mathrm{C}$. The line and the dashed lines represent the fitting of the Arrhenius dependence ie exponential relationship $\left(A_{w}=1-\exp \left(-\frac{\alpha}{c-C_{0}}\right)\right.$ ) to the experimental data. The parameters of the model are summarized in table 2. 


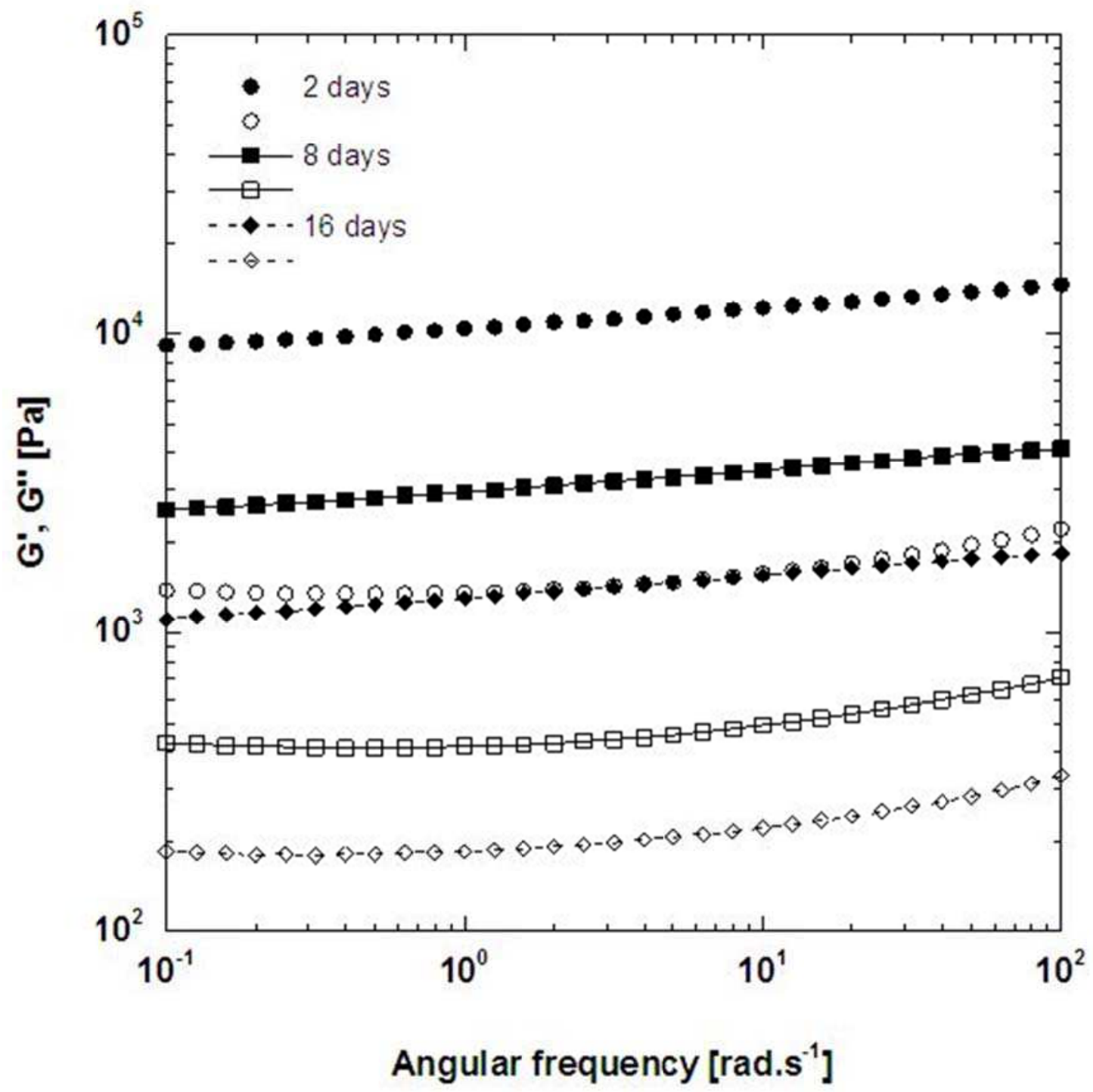

Figure 5: Mechanical spectra of $\mathbf{1 0 . 4 8 \%} \mathrm{w} / \mathrm{w}$ raw sewage sludge at various ageing time. Measurement temperature, $2^{\circ} \mathrm{C}$; strain, $0.3 \%$; time of rest before measurement, $10 \mathrm{~min}$. G', filled symbols; G', empty symbols. 


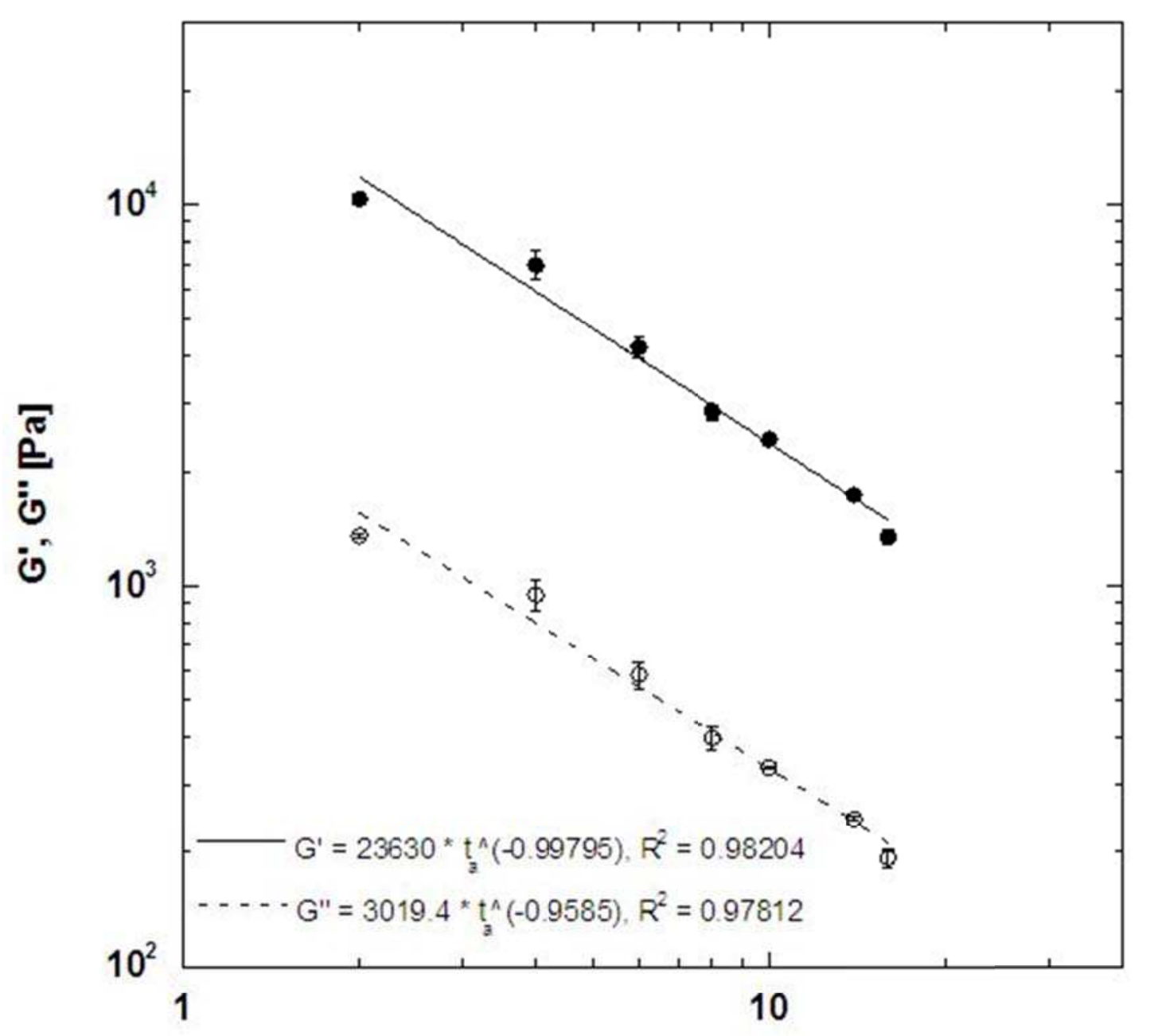

\section{Ageing time, $\mathrm{t}_{\mathrm{a}}$ [day]}

Figure 6: Storage modulus $G^{\prime}(O)$ and loss modulus $G^{\prime \prime}(O)$ of $10.48 \%$ w/w raw sewage sludge as a function of ageing time at $20^{\circ} \mathrm{C}$ and at an angular frequency of $1 \mathrm{rad}^{-1}{ }^{-1}$. The line and the dashed lines represent the fitting of the power-law equation to the experimental data. 


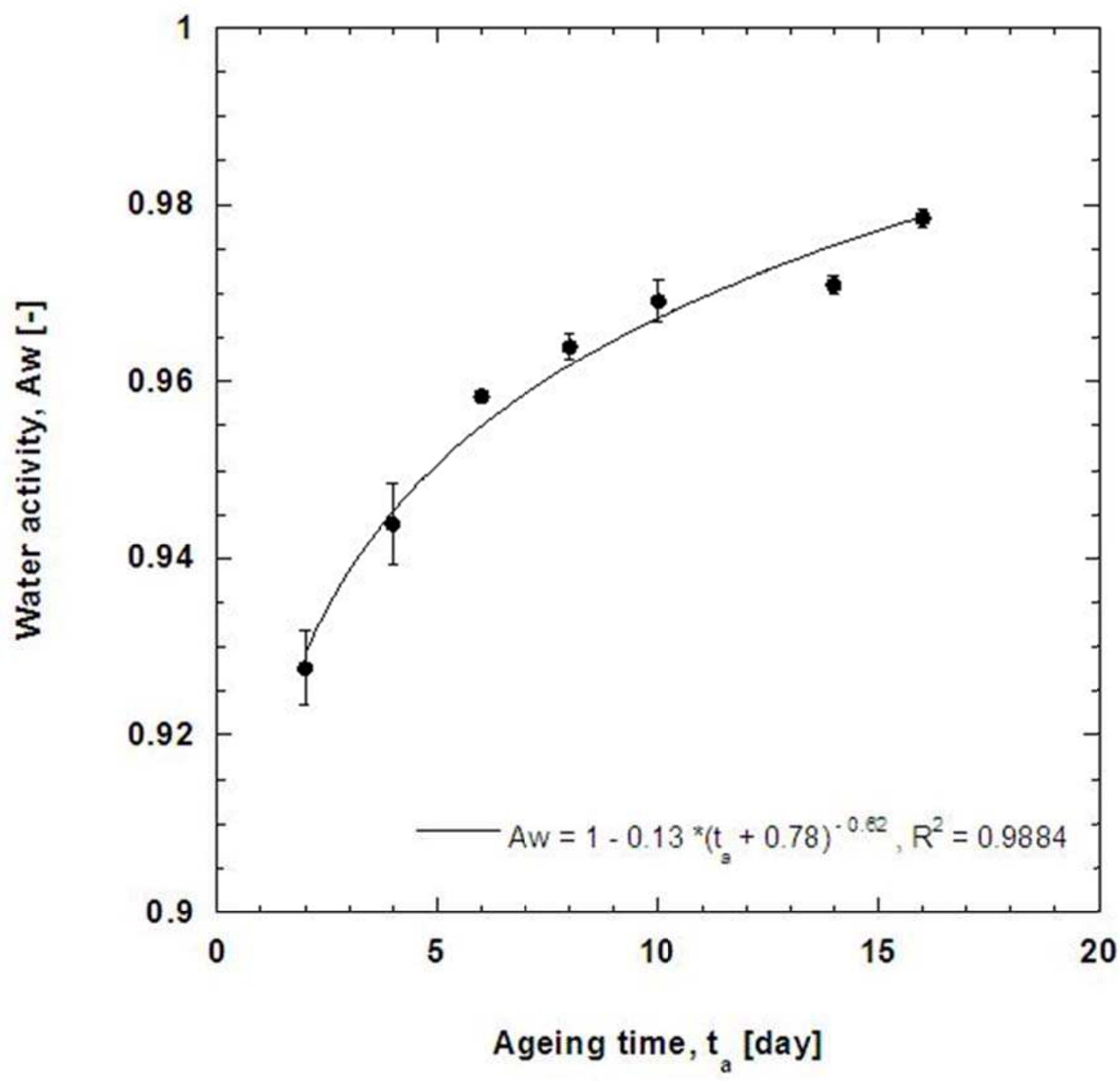

Figure 7: Water activity $A_{w}(O)$ of $10.48 \%$ w/w raw sewage sludge as a function of ageing time at $20^{\circ} \mathrm{C}$. The line represent the fitting of the power-law equation to the experimental data. 

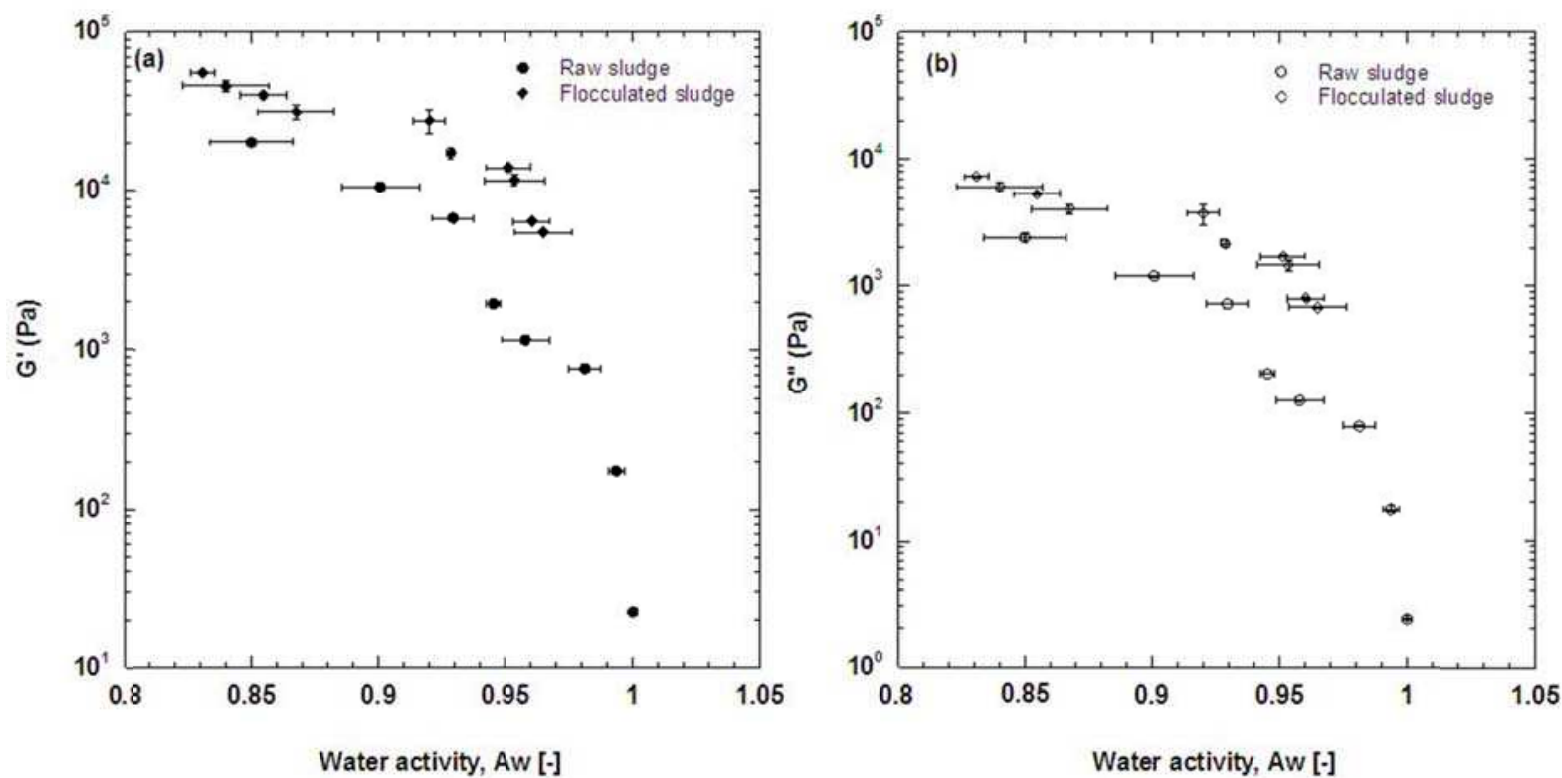

Figure 8: Storage modulus G' (a) and loss modulus G',(b) of raw and flocculated sludge as a function of

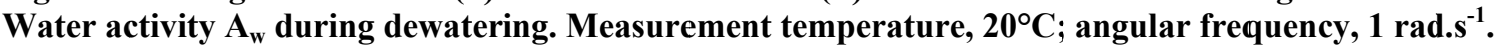




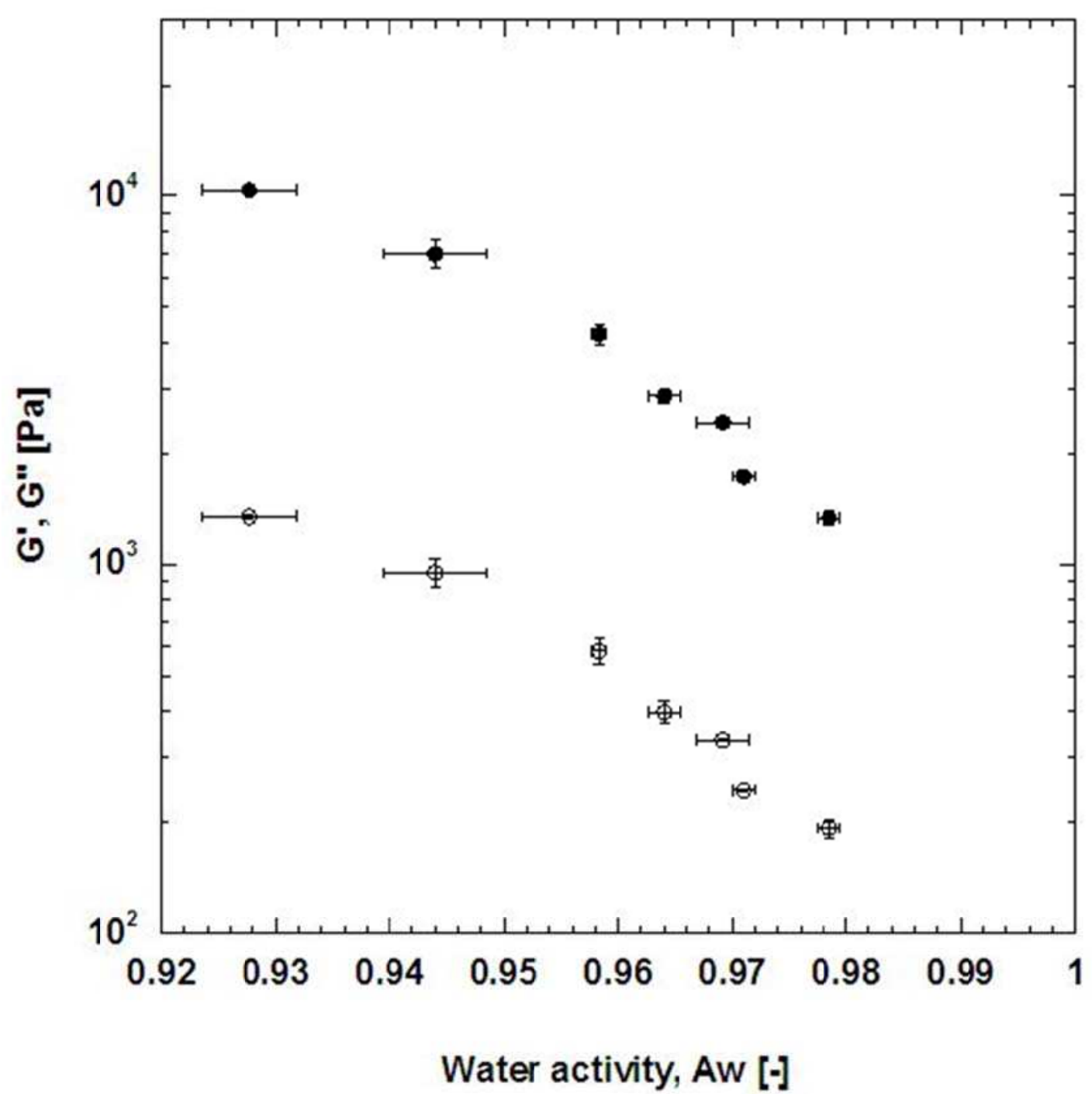

Figure 9: Storage modulus G' $(O)$ and loss modulus $G^{\prime},(O)$ of $10.48 \%$ w/w raw sewage sludge as a function of Water activity Aw during ageing. Measurement temperature, $20^{\circ} \mathrm{C}$; angular frequency, 1 $\operatorname{rad} . s^{-1}$. 